\title{
Analysis of Surgical Results According to the Number of Fused Levels in Anterior Cervical Discectomy and Fusion: A Retrospective Study
}

\author{
Bum-Suk Yu, Farid Yudoyono, Seong Yi, Yoon Ha, Keung Nyun Kim, Do Heum Yoon, Dong Ah Shin \\ Department of Neurosurgery, Severance Hospital, Yonsei University College of Medicine, Seoul, Republic of Korea
}

Corresponding author:

Dong Ah Shin

Department of Neurosurgery,

Yonsei University College of

Medicine, 50 Yonsei-ro,

Seodaemun-gu, Seoul 03722,

Republic of Korea

Tel: $+82-2-2228-2150$

Fax: +82-2-393-9979

E-mail: shindongah@me.com

Received: July 9, 2017

Revised: July 31, 2017

Accepted: August 1, 2017
Objective: To analyze the surgical outcomes of patients who underwent 1-level, 2-level, and 3-level anterior cervical discectomy and fusion (ACDF). Methods: We retrospectively assessed 182 patients with symptomatic cervical degenerative disease who underwent ACDF with allograft and a plate at our institution between January 2012 and March 2014. After only including patients with a $>24$ month follow-up, 142 fulfilled our inclusion criteria (71 underwent 1-level, 46 underwent 2- level, and 25 underwent 3-level ACDF). The assessment tools included the Short Form-36 (SF-36), Neck Disability Index (NDI), and visual analog scale (VAS). The fusion status was measured by radiography at 24 months postoperatively. Statistical analyses were performed with SPSS version 20.0. Results: The fusion rate was $84 \%$ in the 3 -level group, $98.6 \%$ in the 1 -level group, and $97.8 \%$ in the 2 -level $(p=0.017)$. There was a significant difference between 2-level vs. 3-level ACDF $(p=0.049)$. Complications occurred in 4 (16\%) patients of the 3-level group, 2 (2.8\%) patients of the 1-level group, and $1(2.2 \%)$ patient of the 2 -level group $(\mathrm{p}=0.030)$. The subsidence rate was significantly higher in the 3 -level group than in the other 2 groups (52\%, $p=0.047)$. Significant differences were observed between groups in terms of the NDI and SF-36 at the last follow-up ( $p=0.040$ and $p=0.046$, respectively). Conclusion: The surgical outcome of the 3-level ACDF group was worse than that of the 1level or 2-level ACDF groups, which was indicated by the lower fusion rate, higher complication rate, and higher subsidence rate.

Key Words: Spinal fusion; Arthrodesis; Cervical vertebrae; Treatment outcome

\section{INTRODUCTION}

Anterior cervical discectomy and fusion (ACDF) has been the gold standard treatment for degenerative cervical diseases since its introduction by Smith and Robinson ${ }^{2}$. Most surgeons believe that ACDF represents the surgical standard of care. This technique is widely used to treat various diseases, such as traumatic cord injuries, degenerative radiculopathy, and myelopathy ${ }^{2}$. In addition, clinical and radiographic outcomes following ACDF have been well established in the literature ${ }^{13)}$. Remarkable studies have reported good fusion rates and excellent clinical outcomes ${ }^{11,13)}$.

However, several studies have reported higher frequencies of overall complications and reoperation rates of ACDF procedures, especially in patients with multilevel diseases, ${ }^{5,6,12)}$. Veeravagu et al. ${ }^{13)}$ reported that patients who underwent greater than 2-level ACDF were more likely to require revision than those who with two-level ACDF or less. Similarly, the risk of any complication or the need for readmission was also greater in patients who underwent greater than 2-level ACDF procedures ${ }^{12)}$

According to previous studies and our experience, we assumed that unlike 1-level or 2-level ACDF, there would be a clear difference in 3-level ACDF. However, to the best of our knowledge, no study has compared 3-level ACDF with 2-level and 1-level ACDF. Therefore, we investigated the demographic characteristics, clinical outcomes, complications, revision rates, fusion rates, and subsidence rates in patients undergoing 1-level to 3-level ACDF procedures with a minimum 2year follow-up. The purpose of this study was to analyze the surgical outcomes of patients who underwent 1-level, 2-level, and 3-level ACDF.

\section{MATERIALS AND METHODS}

\section{Subjects}

We retrospectively assessed 182 patients with symptomatic 
cervical degenerative disease who underwent ACDF with a cage-plate device from January 2012 to March 2014 at our institution. Informed consent was obtained or waived from all patient. Patients were divided into 3 groups: 1-level, 2-level, and 3-level ACDF. Inclusion criterion was patients older than 18 years undergoing 1-level, 2-level, or 3-level ACDF with interbody grafting with allograft and anterior cervical plating. Exclusion criteria were (1) patients with a history of previous cervical spine operation (e.g., ACDF with autograft or a cage, a procedure due to cervical trauma, spinal cord tumor resection, or primary spondylodiscitis, anterior cervical corpectomy, and combined surgery) ( $\mathrm{n}=5)$; (2) patients diagnosed as having ossification of the posterior longitudinal ligament $(n=15)$; and (3) patients with less than 24 months of follow-up $(n=10)$.

Demographic characteristics and comorbidities of all patients were collected by medical record review. Demographic characteristics of interest included age, sex, comorbidities, and presenting symptoms. Comorbidities of interest were the presence of hypertension, diabetes, heart disease, kidney disease, thyroid disease, and liver disease or brain disease.

A standard Smith-Robinson approach was used to access the disc space, and typical discectomy, decompression of neural structures, and removal of osteophytes was performed.

\section{Outcome Assessment}

Outcome variables were obtained from clinic notes at 3, 12 , and 24 months and at the last available follow-up for each patient. Measured outcomes included the Neck Disability Index (NDI), visual analog scale (VAS) for neck and arm pain, and Short Form-36 (SF-36)-item health survey. Mental composite score (MCS) and Physical composite score (PCS). A mean change in the scores was determined from the most symptomatic arm preoperatively through the last follow-up. Intraoperative data, including the fusion levels, operation time (hr), and estimated blood loss (EBL) (cc), were collected from the operative records.

\section{Radiological Assessment}

Bony fusion was assessed at follow-up visits (3, 12, and 24 months) postoperatively through a combination of conventional radiographs and 3-dimensional computed tomography scans. Visualization of trabecular bridging on the bone-graft interface and the absence of radiolucent gaps between the endplate and graft were assessed. Subsidence was assessed by comparing radiographs immediately postoperatively (within 1 week) with those at the final follow-up. The subsidence rate was defined as a decrease in $2 \mathrm{~mm}$ or more of the superior and inferior parts of the cage into the vertebral body from that measured on the postoperative radiograph. Adjacent segment disease (ASD) was defined as clinically symptomatic radiographic degeneration of the adjacent segment.

\section{Statistical Analysis}

One-way analysis of variance was used to identify a significant difference in variables among the 3 groups. Post hoc analysis among the 3 groups was performed using the Bonferroni corrected p-value. Cross tabulation tables were analyzed using the Fisher exact and Pearson chi-square test. A p-value <0.05 was used a threshold for statistical significance. All statistical analyses were performed using SPSS version 20.0 (IBM Corp., Armonk, NY, USA).

\section{RESULTS}

\section{Demographic Characteristics}

Sex, age, comorbidities, and presenting symptoms were similar among the 1-level, 2-level, and 3-level ACDF groups (Table 1). The average duration of follow-up for all the groups was slightly more than 2 years. There was a significant difference in the treated level, because the number of levels included in the 3-level ACDF group was high.

\section{Surgical Outcomes}

Compared with 2-level and 3-level ACDF, 1-level ACDF was associated with a statistically significantly lower operative time and EBL (Table 2). Additionally, 2-level ACDF was associated with a significantly lower operative time and EBL than 3-level $\mathrm{ACDF}$. The length of hospital stay was also significantly different among the 3 groups. A longer ACDF procedure resulted in a higher EBL and longer length of hospital stay. Complications occurred in 4 patients of the 3-level ACDF group, 2 patients of the 1-level ACDF group, and 1 patient of the 2-level ACDF group. Major complications (i.e., screw pullout and deep wound infection) occurred in 2 patients in the 3-level ACDF group; minor complications occurred in the other 2 groups (Fig. 1). Overall, complications were significantly highest in the 3-level ACDF group (16\%; p=0.03).

The preoperative and last follow-up VAS scores were not significantly different among the 3 groups $(\mathrm{p}=0.698)$ (Table 3$)$. The mean scores improved significantly by the last follow-up in all groups. The mean difference in the VAS score between the preoperative period and last follow-up was highest in the 2-level ACDF group (-5.2 $\pm 2.1, \mathrm{p}<0.05)$.

Preoperative NDI scores were not significantly different among the 1-level, 2-level, and 3-level ACDF groups (28.8, 26.8, and 27.0 , respectively; $\mathrm{p}=0.71$ ). At the last follow-up, the NDI score improved significantly in all groups $(\mathrm{p}<0.001)$. There was no significant difference in the NDI score at the last follow-up between the 1-level ACDF group and 2-level ACDF group ( $\mathrm{p}=$ 0.390 ). However, there was a significant difference between the 2-level ACDF group and 3-level ACDF group ( $\mathrm{p}=0.005)$, and 
between the 1-level ACDF group and 3-level ACDF group ( $\mathrm{p}=$ 0.04 ) at the last follow-up. The mean difference in the NDI score between the preoperative and last follow-up was lower in the 3-level ACDF group than in the other 2 groups. Our results showed that SF-36 PCSs in the 1-level ACDF group increased from 56.6 to 67.6, and these scores in the 2-level ACDF group increased from 53.7 to 63.5. The SF-36 PCS in the 3-level ACDF group increased from 59.0 to 62.0, but this was not significant improvement. However, 1-level and 2-level ACDF groups had a significant improvement in the SF-36 PCS at the last follow-up $(\mathrm{p}<0.001)$. There was a significant difference in the SF-36 PCSs among the 3 groups at the last follow-up. Moreover, SF-36 MCSs for patients who underwent 1-level ACDF increased from 54.9 to 63.6, whereas these scores for patients who underwent 2-level ACDF increased from 54.8 to 60.6.

The SF-36 MCS for 3-level ACDF increased from 58.8 to
61.9, but this was not significant improvement. There was no difference in the amount of improvement from the preoperative SF36-MCS/PCS scores reported by patients who underwent 3-level ACDF.

\section{Radiological Outcome}

There were 19 patients (26.8\%) in the 1-level ACDF group, $12(26.1 \%)$ in the 2-level ACDF group, and $13(52 \%)$ in the 3-level ACDF group with subsidence (Table 4, Fig. 2). There were significant differences between the groups $(\mathrm{p}=0.047)$ (Table 4). The fusion rates were $98.6 \%$ for 1-level ACDF, $97.8 \%$ for 2-level ACDF, and 84.0\% for 3-level ACDF ( $\mathrm{p}=0.017$, Fisher exact test). There were significant differences among the groups. ASD was assessed separately at the superior and inferior levels adjacent to the operated segment. There were 3 patients (4.2\%)

Table 1. Preoperative data

\begin{tabular}{|c|c|c|c|c|}
\hline Parameter & 1-level ACDF $(n=71)$ & 2-level ACDF $(n=46)$ & 3-level ACDF $(n=25)$ & Overall p-value \\
\hline Follow-up duration (years) & $2.1 \pm 0.5$ & $2.2 \pm 0.5$ & $2.0 \pm 0.4$ & 0.390 \\
\hline Mean age (years) & $51.05 \pm 11.45$ & $52.24 \pm 12.15$ & $51.56 \pm 10.51$ & 0.870 \\
\hline Sex (male) & $30(42.3 \%)$ & $13(28.3 \%)$ & $6(24.0 \%)$ & 0.142 \\
\hline Comorbidities & $25(35.2 \%)$ & 19 (41.3\%) & $6(24.0 \%)$ & 0.345 \\
\hline \multicolumn{5}{|l|}{ Symptoms } \\
\hline Radiculopathy & $51(71.8 \%)$ & $32(69.6 \%)$ & $19(76.0 \%)$ & 0.145 \\
\hline Myelopathy & $20(28.2 \%)$ & $14(30.4 \%)$ & $6(24.0 \%)$ & 0.210 \\
\hline Weakness & $11(15.5 \%)$ & $5(10.9 \%)$ & $2(8.0 \%)$ & 0.320 \\
\hline Sensory change & $15(21.1 \%)$ & $8(17.4 \%)$ & $5(20.0 \%)$ & 0.342 \\
\hline Pain & 49 (69.0\%) & $38(82.6 \%)$ & $21(84.0 \%)$ & 0.060 \\
\hline \multicolumn{5}{|l|}{ Treated levels } \\
\hline C3-4 & $9(12.7 \%)$ & $1(2.2 \%)$ & $6(24.0 \%)$ & 0.018 \\
\hline C4-5 & $8(11.3 \%)$ & 24 (52.2\%) & 25 (100.0\%) & $<0.001$ \\
\hline C5-6 & $34(47.9 \%)$ & $45(97.8 \%)$ & $25(100.0 \%)$ & $<0.001$ \\
\hline C6-7 & $20(28.2 \%)$ & 22 (47.8\%) & 19 (76.0\%) & $<0.001$ \\
\hline
\end{tabular}

ACDF: anterior cervical discectomy and fusion.

Table 2. Perioperative data

\begin{tabular}{|c|c|c|c|c|c|}
\hline Parameter & $\begin{array}{c}\text { 1-level ACDF } \\
(n=71)\end{array}$ & $\begin{array}{c}\text { 2-level ACDF } \\
(n=46)\end{array}$ & $\begin{array}{c}\text { 3-level ACDF } \\
(n=25)\end{array}$ & $\begin{array}{l}\text { Overall } \\
\text { p-value }\end{array}$ & $\begin{array}{l}\text { Post hoc } \\
\text { analysis }\end{array}$ \\
\hline Mean estimated blood loss (cc) & $128.5 \pm 112.7$ & $180.4 \pm 119.9$ & $227.2 \pm 131.2$ & 0.001 & $1<2<3$ \\
\hline Mean operative time (days) & $1.7 \pm 0.6$ & $2.5 \pm 0.7$ & $3.1 \pm 0.4$ & $<0.0001$ & $1<2<3$ \\
\hline Mean length of stay (days) & $4.9 \pm 1.1$ & $5.8 \pm 2.6$ & $5.3 \pm 0.9$ & 0.01 & $1<2<3$ \\
\hline Complications & $2(2.8 \%)$ & $1(2.2 \%)$ & $4(16.0 \%)$ & & \\
\hline Screw pullout & 0 & 0 & 1 & & \\
\hline Hoarseness & 0 & 1 & 2 & 0.03 & 1 or $2<3$ \\
\hline Infection & 0 & 0 & 1 & & \\
\hline Weakness & 2 & 0 & 0 & & \\
\hline
\end{tabular}

ACDF: anterior cervical discectomy and fusion. 
Table 3. Clinical outcomes

\begin{tabular}{|c|c|c|c|c|c|}
\hline Parameter & 1-level ACDF $(n=71)$ & 2-level ACDF $(n=46)$ & 3-level ACDF $(n=25)$ & Overall $p$-value & Post hoc analysis \\
\hline \multicolumn{6}{|l|}{ NDI } \\
\hline Preoperative & $28.8 \pm 15.4$ & $26.8 \pm 11.6$ & $27.0 \pm 11.5$ & 0.71 & \\
\hline Last follow-up & $16.2 \pm 11.0$ & $14.7 \pm 8.8$ & $20.9 \pm 8.7$ & 0.04 & 1 or $2<3$ \\
\hline Difference & $12.6 \pm 16.1$ & $12.2 \pm 14$ & $6.1 \pm 8.8$ & 0.14 & \\
\hline p-value & $<0.001$ & $<0.001$ & 0.002 & & \\
\hline \multicolumn{6}{|l|}{ SF-36 PCS } \\
\hline Preoperative & $56.6 \pm 9.4$ & $53.7 \pm 9.6$ & $59.0 \pm 10.3$ & 0.076 & \\
\hline Last follow-up & $67.6 \pm 10.7$ & $63.5 \pm 12.1$ & $62.0 \pm 11.6$ & 0.046 & \\
\hline Difference & $10.9 \pm 10.9$ & $9.7 \pm 13.9$ & $2.9 \pm 9.1$ & 0.020 & 1 or $2>3$ \\
\hline $\mathrm{p}$-value & $<0.001$ & $<0.001$ & 0.100 & & \\
\hline \multicolumn{6}{|l|}{ SF-36 MCS } \\
\hline Preoperative & $54.9 \pm 9.1$ & $54.8 \pm 9.8$ & $58.8 \pm 10.5$ & 0.155 & \\
\hline Last follow-up & $63.6 \pm 10.9$ & $60.6 \pm 13.0$ & $61.9 \pm 11.6$ & 0.392 & \\
\hline Difference & $8.7 \pm 12.7$ & $5.7 \pm 15.0$ & $3.0 \pm 8.9$ & 0.146 & \\
\hline $\mathrm{p}$-value & $<0.001$ & 0.012 & 0.100 & & \\
\hline \multicolumn{6}{|l|}{ VAS score } \\
\hline Preoperative & $6.4 \pm 0.7$ & $6.6 \pm 2.1$ & $6.6 \pm 1.1$ & 0.698 & \\
\hline Last follow-up & $1.7 \pm 1.1$ & $1.4 \pm 0.7$ & $1.9 \pm 0.6$ & 0.059 & \\
\hline Difference & $4.6 \pm 1.2$ & $5.2 \pm 2.1$ & $4.7 \pm 1.1$ & 0.174 & \\
\hline p-value & $<0.001$ & $<0.001$ & $<0.001$ & & \\
\hline
\end{tabular}

ACDF: anterior cervical discectomy and fusion; NDI: Neck Disability Index; SF-36: Short-Form-36; PCS: physical composite score; MCS: mental composite score; VAS: visual analog scale.
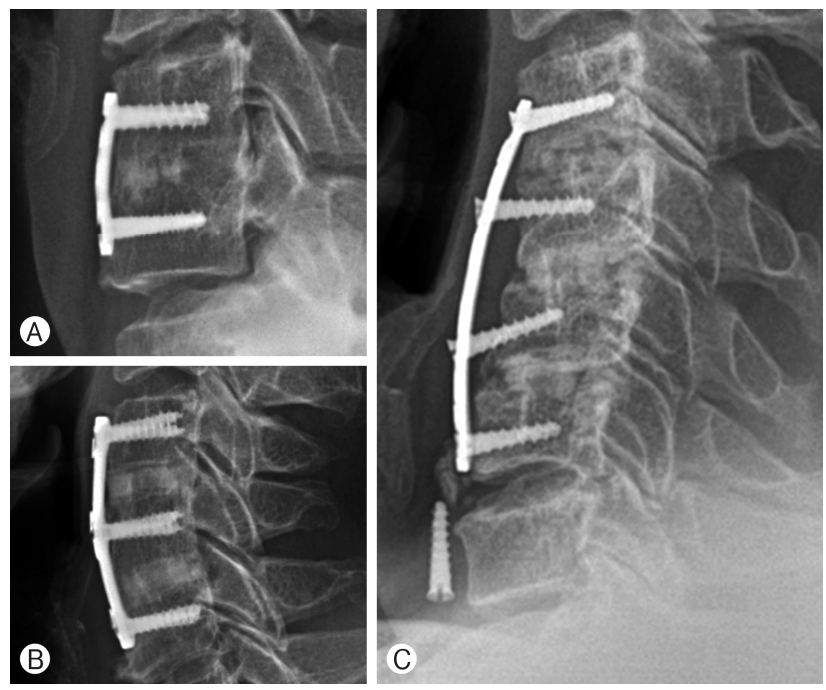

Fig. 1. Lateral view of the cervical spine obtained at the 1-year follow-up visit. (A) Case example of 1-level anterior cervical discectomy and fusion (ACDF). (B) Case example of 2-level ACDF. (C) A 55-year old woman underwent 3-level ACDF. One-year postoperatively, a screw was pulled out, and she underwent revision operation.

in the 1-level ACDF group, $4(8.7 \%)$ in the 2-level ACDF group, and $4(16 \%)$ in the 3 -level ACDF group with ASD; this was not
Table 4. Postoperative outcomes

\begin{tabular}{|c|c|c|c|c|}
\hline Parameter & $\begin{array}{l}\text { 1-level ACDF } \\
(n=71)\end{array}$ & $\begin{array}{c}\text { 2-level ACDF } \\
(n=46)\end{array}$ & $\begin{array}{c}\text { 3-level ACDF } \\
(n=25)\end{array}$ & $\begin{array}{l}\text { Overall } \\
\text { p-value }\end{array}$ \\
\hline Fusion rate & 70 (98.6\%) & 45 (97.8\%) & 21 (84.0\%) & 0.017 \\
\hline Subsidence rate & 19 (26.8\%) & 12 (26.1\%) & 13 (52.0\%) & 0.047 \\
\hline ASD & 3 (4.2\%) & 4 (8.7\%) & 4 (16.0\%) & 0.120 \\
\hline Reoperation & $1(1.4 \%)$ & $0(0.0 \%)$ & $1(4.0 \%)$ & 0.430 \\
\hline
\end{tabular}

significantly different $(\mathrm{p}=0.12)$. Only 1 patient with ASD after 1-level ACDF underwent reoperation. The other 10 patients with ASD required only conservative care.

\section{DISCUSSION}

This study created a unique opportunity to compare outcomes of patients undergoing 1-level vs. 2-level vs. 3-level ACDF with 2-year follow-up. The comparison of demographic characteristics found that the 3 groups were similar. Significant difference in the quality of life or incidence of complications was reported between the patients. The fusion rate and sub- 


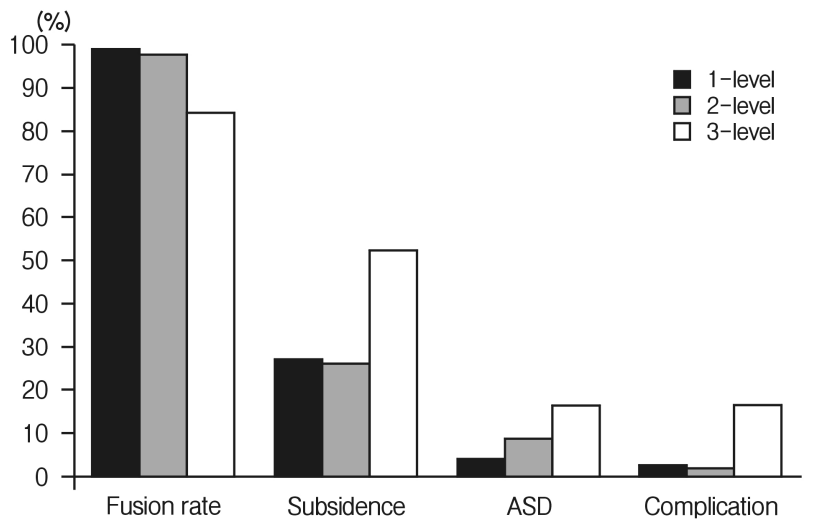

Fig. 2. Surgical outcomes for all groups. There is a significantly high incidence of complications and high subsidence rate in the 3-level anterior cervical discectomy and fusion (ACDF) group. A significantly lower fusion rate is observed in the 3-level ACDF group. ASD: adjacent segment disease.

sidence were correlated with fusion level. Three level ACDF revealed worse surgical outcomes and higher complication. Otherwise, there was no statistically significant difference between 1-level and 2-level ACDF groups on any of the outcome measures, including the NDI, VAS pain scores, SF-36, and reoperations

These findings are similar to those of a previous study that reported these outcome measures for 1-level and 2-level $\mathrm{ACDF}^{11)}$. As in the current study, statistically significant improvements were noted throughout the follow-up in the 1-level and 2-level groups ${ }^{11)}$. There was no statistically significant difference between 1-level and 2-level ACDF groups in any of the outcome measures, including the NDI, VAS pain score, SF-36, and occurrence of reoperations ${ }^{11)}$. However, in multilevel discectomy, the success rate decreases as the number of levels increase. Some literature supports consistent rates of $10 \%$ to $12 \%$ non-fusion for 1-level anterior discectomy and bone fusion, 20\% to $27 \%$ for 2-level, and approximately 30\% to 56\% for 3-level fusions ${ }^{4}$. One study reported that at the 2-year follow-up, fusion rates were $89.3 \%$ in the 1-level fusion group and $79.8 \%$ in the 2-level fusion group". Another study reported that the risk of complications or the need for reoperation was also greater in patients who underwent more than 2-level $\mathrm{ACDF}^{12)}$.

In previous studies, fusion rates after 3-level ACDF were generally $\operatorname{low}^{2,11,13)}$. As high rates of pseudarthrosis have been observed after 3-level ACDF, some authors have recommended corpectomy and strut grafting in multilevel cases ${ }^{8)}$. This may be because of the increased number of graft-bone interfaces, as well as the altered biomechanics and increasing contact stress at graft-bone interfaces when the number of operative levels increases. Brodke and Zdeblick ${ }^{2)}$ reported a clinically significant pseudarthrosis rate of $17 \%$. Wang et al. ${ }^{14)}$ found that $18 \%(7 / 40)$ of patients had pseudarthrosis after 3-level plated ACDF. Bolesta et al. ${ }^{1)}$ performed a prospective study of $15 \mathrm{pa}$ - tients who underwent three operative levels, and they reported the highest nonunion rate in 8 of 15 patients (53\%). Emery et al. ${ }^{7)}$ reported the pseudarthrosis rate for non-plated 3-level discectomies as $44 \%$. The fusion rate in our study was $84 \%$, which is similar to the rate reported by Wang et al. ${ }^{14)}$. Nevertheless, this fusion rate remains lower than the rate for 1-level or 2-level ACDF, which ranges from $96 \%$ to $100 \%$,

Some studies did not show a significant difference in the clinical outcome and union rate when comparing 3-level and 2-level with 1-level ACDF. Lin et al. ${ }^{10)}$ analyzed dynamic changes and clinical outcomes following ACDF from 1-level, 2-level, and 3-level cervical fusion with only cages, no plates. There was no significant difference with respect to the postoperative VAS score for neck pain and fusion rate in the three surgical groups. However, since the study only involved patients with cages, the results would not be consistent with our study. ASD is a well-known sequela of cervical fusion, and various etiologies have been reported, including natural disease progression vs. postoperative biomechanical alterations of the spine ${ }^{33}$. Additionally, there are conflicting data on whether the rate of ASD is higher in single vs. multilevel fusion. In a clinical study, Bydon et al. ${ }^{3}$ ) demonstrated that the rates of ASD were not significantly different in patients undergoing 1-level, 2-level, or 3-level ACDF ( $\mathrm{p}=0.910)$. Conversely, Prasarn et al. ${ }^{12)}$ found that the rate of ASD was significantly higher after 2-level fusion than after 1-level fusion. However, in the present study, the number of patients who developed symptomatic ASD was highest in the 3-level ACDF group (16\%), but this was not significantly different compared with the other 2 groups $(\mathrm{p}=0.12)$. Furthermore, 4 patients in the 3-level ACDF group with radiographic ASD did not require reoperation.

This study has several limitations, including a short follow-up period of 1 year and a small number of patients. Specific complications, such as dysphagia, were assessed based on a surgeon's clinical assessment, not on other more objective measures, such as swallowing evaluations and/or direct laryngoscopy. Additionally, there is also a risk of information bias, as with all retrospective studies based on medical chart or operative note reviews. The global sagittal balance and other radiographic measures were unavailable for review in all patients, and this information may have contributed to understanding the overall outcomes. Prospective studies are most desired for this type of clinical study, and future research on the longterm outcomes of ACDF, such as the reoperation rates, patientreported outcomes, and complications, etc., are needed.

\section{CONCLUSION}

The surgical outcome of the 3-level ACDF group was worse than that of the 1-level and 2-level ACDF group, as indicated by the lower fusion rate, higher complication rate, and higher subsidence rate. 


\section{ACKNOWLEDGEMENT}

This work was supported by the Industrial R\&D Program of MOTIE/KEIT (10052732).

\section{REFERENCES}

1. Bolesta MJ, Rechtine GR, 2nd, Chrin AM: Three- and four-level anterior cervical discectomy and fusion with plate fixation: a prospective study. Spine (Phila Pa 1976) 25:2040-2044; discussion 2045-2046, 2000

2. Brodke DS, Zdeblick TA: Modified Smith-Robinson procedure for anterior cervical discectomy and fusion. Spine (Phila Pa 1976) 17:S427-S430, 1992

3. Bydon M, Xu R, De la Garza-Ramos R, Macki M, Sciubba DM, Wolinsky JP, et al.: Adjacent segment disease after anterior cervical discectomy and fusion: Incidence and clinical outcomes of patients requiring anterior versus posterior repeat cervical fusion. Surg Neurol Int 5:S74-S78, 2014

4. Cho DY, Lee WY, Sheu PC: Treatment of multilevel cervical fusion with cages. Surg Neurol 62:378-385, discussion 385-376, 2004

5. De Palma AF, Cooke AJ: Results of anterior interbody fusion of the cervical spine. Clin Orthop Relat Res 60:169-185, 1968

6. Emery SE, Bolesta MJ, Banks MA, Jones PK: Robinson anterior cervical fusion comparison of the standard and modified techniques. Spine (Phila Pa 1976) 19:660-663, 1994
7. Emery SE, Fisher JR, Bohlman HH: Three-level anterior cervical discectomy and fusion: radiographic and clinical results. Spine (Phila Pa 1976) 22:2622-2624; discussion 2625, 1997

8. Grossman W, Peppelman WC, Baum JA, Kraus DR: The use of freeze-dried fibular allograft in anterior cervical fusion. Spine (Phila Pa 1976) 17:565-569, 1992

9. Hilibrand AS, Carlson GD, Palumbo MA, Jones PK, Bohlman $\mathrm{HH}$ : Radiculopathy and myelopathy at segments adjacent to the site of a previous anterior cervical arthrodesis. J Bone Joint Surg Am 81:519-528, 1999

10. Lin HL, Cho DY, Liu YF, Lee WY, Lee HC, Chen CC: Change of cervical balance following single to multi-level interbody fusion with cage. Br J Neurosurg 22:758-763, 2008

11. Papadopoulos EC, Huang RC, Girardi FP, Synnott K, Cammisa FP, Jr.: Three-level anterior cervical discectomy and fusion with plate fixation: radiographic and clinical results. Spine (Phila Pa 1976) 31:897-902, 2006

12. Prasarn ML, Baria D, Milne E, Latta L, Sukovich W: Adjacentlevel biomechanics after single versus multilevel cervical spine fusion. J Neurosurg Spine 16:172-177, 2012

13. Veeravagu A, Cole T, Jiang B, Ratliff JK: Revision rates and complication incidence in single- and multilevel anterior cervical discectomy and fusion procedures: an administrative database study. Spine J 14:1125-1131, 2014

14. Wang JC, McDonough PW, Kanim LE, Endow KK, Delamarter RB: Increased fusion rates with cervical plating for three-level anterior cervical discectomy and fusion. Spine (Phila Pa 1976) 26:643-646; discussion 646-647, 2001 